\title{
Analysis of novel domain-specific mutations in the zebrafish ndr2/cyclops gene generated using CRISPR-Cas9 RNPs
}

Ashley N. Turner ${ }^{1,2}$, Reagan S. Andersen ${ }^{3}$, Ivy E. Bookout ${ }^{3}$, Lauren N. Brashear ${ }^{3}$, James C. Davis $^{3}$, David M. Gahan ${ }^{3}$, John P. Gotham ${ }^{3}$, Baraa A. Hijaz ${ }^{3}$, Ashish S. Kaushik ${ }^{3}$, Jordan B McGill ${ }^{3}$, Victoria L. Miller ${ }^{3}$, Zachariah P. Moseley ${ }^{3}$, Cerissa L. Nowell ${ }^{3}$, Riddhi K. Patel ${ }^{3}$, Mia C. Rodgers ${ }^{3}$, Yazen A. Shihab ${ }^{3}$, Austin P. Walker ${ }^{3}$, Sarah R. Glover ${ }^{3}$, Samantha D. Foster ${ }^{3}$, Anil K. Challa ${ }^{1}$

1. Department of Genetics, 2. Graduate Biomedical Sciences Program (Genetics, Genomics \& Bioinformatics Theme), 3. Science \& Technology Honors Program (all undergraduate students contributed equally in a Course-based Undergraduate Research Experience)

Corresponding author:

Anil Kumar Challa, PhD

Address:

Department of Genetics, University of Alabama at Birmingham Hugh Kaul Human Genetics Building, Rm 624

720 20th Street South

Birmingham, AL 35294

United States of America

Keywords:

zebrafish, ndr2, cyclops, CRISPR-Cas9, RNP complex, RNP 


\section{Abstract}

Nodal-related protein (ndr2) is a member of the transforming growth factor type $\beta$ superfamily of factors and is required for ventral midline patterning of the embryonic central nervous system in zebrafish. In humans, mutations in the gene encoding nodal cause holoprosencephaly and heterotaxy. Mutations in the $n d r 2$ gene in the zebrafish (Danio rerio) lead to similar phenotypes, including loss of the medial floor plate, severe deficits in ventral forebrain development, and cyclopia. Alleles of the $n d r 2$ gene have been useful in studying patterning of ventral structures of the central nervous system. Fifteen different $n d r 2$ alleles have been reported in zebrafish, of which eight were generated using chemical mutagenesis, four were radiation-induced, and the remaining alleles were obtained via random insertion, gene targeting (TALEN), or unknown methods. Therefore, most mutation sites were random and could not be predicted a priori. Using the CRISPR-Cas9 system from Streptococcus pyogenes, we targeted distinct regions in all three exons of zebrafish ndr2 and observed cyclopia in the injected $\left(\mathrm{G}_{0}\right)$ embryos. We show that the use of sgRNA-Cas9 ribonucleoprotein (RNP) complexes can cause penetrant cyclopic phenotypes in injected $\left(\mathrm{G}_{0}\right)$ embryos. Targeted PCR amplicon analysis using Sanger sequencing showed that most of the alleles had small indels resulting in frameshifts. The sequence information correlates with the loss of ndr2 activity. In this study, we validate multiple CRISPR targets using an in vitro nuclease assay and in vivo analysis using embryos. We describe one specific mutant allele resulting in loss of conserved terminal cysteine-coding sequences. This study is another demonstration of the utility of the CRISPR-Cas9 system in generating domainspecific mutations and provides further insights into the structure-function of the $n d r 2$ gene. 


\section{Introduction}

The transforming growth factor $\beta$ (TGF- $\beta$ ) superfamily is one of the major groups of secreted signaling molecules that is important in cell-to-cell communication and coordinating pattern formation during development $[1,2]$. The Nodal proteins of the TGF- $\beta$ superfamily have been found in every vertebrate examined to date. Nodal factors play pivotal roles during embryogenesis of chordates and have been implicated in several developmental processes, including mesoderm and endoderm formation, anterior-posterior patterning and left-right axis formation [3-8].

In humans, there are several documented mutations in the NODAL gene that cause holoprosencephaly and heterotaxy [9]. In zebrafish, there are three NODAL-related paralogs (ndr1/squint, ndr2/cyclops, and ndr3/southpaw) that are very similar but have specialized functions. During embryogenesis cyclops (cyc)/nodal related 2 (ndr2) is required for ventral midline patterning of the central nervous system $[3,4,7,8,10,11]$ (henceforth referred only as $n d r 2)$. Mutations in $n d r 2$ in the zebrafish lead to similar phenotypes observed in humans, including loss of medial floor plate, severe deficits in ventral forebrain development, and cyclopia [3, 4, 10, 12-18].

The TGF- $\beta$ proteins, including nodal proteins, are synthesized as precursors and the propeptide is cleaved off by proprotein convertase at a unique cleavage site [19]. These cleavage site sequences vary among TGF- $\beta$ precursors [20]. One conserved feature consists of a dibasic RXXR sequence [1, 19, 21], while other recognition sequences that contain an additional basic residue (RXRXXR, or RXK/RR) are more rapidly cleaved than the minimal $R X X R$ motif $[19,22-24]$. The released C-terminal fragment is the mature ligand and acts as the signaling molecule $[1,19]$. The mature ndr2 ligand has been reported with a range of amino acid sequence lengths, ranging from 119 to 125 , due to variability in the cleavage sequence motif and context of amino acid sequence in this region [3, 4, 21].

There are seven cysteine residues that are conserved in most TGF- $\beta$ family members, six of which interact to form a "cysteine knot" that is essential for biological activity of all TGF- $\beta$ proteins $[25,26]$. The seventh cysteine forms a disulfide bond with a second nodal polypeptide, generating the dimeric form of the ligand that binds to its cognate receptor and leads to activation of downstream signaling pathways [25, 27, 28].

There are 15 previously reported mutations or alleles of zebrafish $n d r 2$ (https://zfin.org/ZDB-GENE-990415-181) (Supplemental Table 1); eight are chemicallyinduced mutations and four are radiation-induced mutations. Phenotypic differences in cyclopia severity have been observed across different alleles. Exact sequence information of the mutant alleles is known only for four of these mutant alleles because all of the above alleles were generated and identified using random mutagenesis and phenotypic screening. 
This has made any attempt at structure-function studies of proteins arising from all the previously generated mutant alleles difficult. Programmable nucleases have become very useful tools for generating mutations in targeted regions of a gene and the genome, and they enable structure-function studies to be carried out with relative ease. We used the CRISPR-Cas9 nuclease system from Streptococcus pyogenes to target distinct regions in all three exons of the zebrafish $n d r 2$ gene to identify additional alleles.

\section{Materials and Methods}

\section{CRISPR/sgRNA design and synthesis}

CRISPR targets were identified in all three exons of the zebrafish ndr2 gene (GRCz10, GCA_000002035.3) using a web-based design tool (Benchling [Biology Software], 2017; Retrieved from https://benchling.com.) (Figure 1). Guides were designed and selected across exons with a range of Doench/Benchling scores (range 3-76.82) provided by Benchling (Figure 1; Supplemental Table 2) [29, 30]. Sixteen guide RNA molecules were generated using a cloning-free method previously described [31]. In brief, dsDNA templates with T7 promoter sequences were generated by annealing gene-specific oligos with a constant/tail oligo followed by a fill-in reaction with T4 DNA polymerase (New England Biology, Ipswich, MA) (Supplemental Table 3) [31]. One or two additional G's were added to the T7 promoter sequence to facilitate efficient transcription (Supplementary Table 3). This product was used as the template for in vitro transcription reactions using the T7 Ampliscribe Kit (Epicenter, Madison, WI). Samples were treated with DNasel and precipitated with ammonium acetate and $100 \%$ ethanol. Air dried samples resuspended in TE buffer were quantified using a Nanodrop UV spectrophotometer and checked for RNA integrity using polyacrylamide gel electrophoresis.

\section{In vitro sgRNA-Cas9 nuclease assay}

In vitro assays for sgRNA-Cas9 nuclease activity were carried out using sgRNAs as previously described [32]. Purified Cas9-NLS protein was obtained from QB3 MacroLab (University of California, Berkeley, CA) and RNP complexes were assembled by combining 10x nuclease buffer (NEBuffer 3.1), Cas9 nuclease (0.1 $\mu \mathrm{L}$ of $2400 \mathrm{ng} / \mathrm{uL}$ ), sgRNA (0.5 $\mu \mathrm{L}$ of $500 \mathrm{ng} / \mathrm{uL}$ ) and incubated at $37^{\circ} \mathrm{C}$ for 15 minutes. PCR amplicons spanning the CRISPRtargeted regions (Supplemental Table 4) were added to the RNP complex and incubated for $37{ }^{\circ} \mathrm{C}$ for one hour followed by RNase treatment at $37{ }^{\circ} \mathrm{C}$ for 45 minutes and protein denaturation at $65{ }^{\circ} \mathrm{C}$ for 10 minutes. This final reaction was analyzed using gel electrophoresis with $6 \%$ polyacrylamide gels. 


\section{Zebrafish breeding and embryo injections}

Zebrafish were housed and maintained at the UAB Zebrafish Research Facility (ZRF). AB strain wild-type zebrafish were used in this study. All experiments were performed in accordance with the recommendations in the Guide for the Care and Use of Laboratory Animals published by the National Institutes of Health. The protocols used were approved by and conducted in compliance with the University of Alabama at Birmingham Institutional Animal Care and Use Committee. Cas9 protein-sgRNA RNPs were co-injected into one-cell stage zebrafish embryos. RNP complexes were formed by combining TE lite (10 mM Tris, $0.1 \mathrm{mM}$ EDTA), Cas9 nuclease $(0.1 \mu \mathrm{L}$ of $2400 \mathrm{ng} / \mathrm{uL})$, sgRNA (0.5 $\mu \mathrm{L}$ of $500 \mathrm{ng} / \mathrm{uL})$, and nuclease-free water and incubated at $37^{\circ} \mathrm{C}$ for 15 minutes. Each embryo was injected with 3-6 $\mathrm{nL}$ of the RNP solution. Dead embryos were removed at mid-gastrulation and the remaining embryos were used for phenotypic analysis and genotyping.

\section{Phenotypic analysis}

Using brightfield light microscopy, 10-20 single injected embryos were examined and embryos showing cyclopia were imaged using a Zeiss Lumar Lamar V12 stereo microscope and AxioVision SE64 Rel.4.9.1 software (Zeiss, Oberkochen, Germany). Embryos were anaesthetized in a solution of embryo medium containing $2 \mathrm{mM}$ tricaine (Sigma-Aldrich, St. Louis, MO) to prevent gross movements during imaging. Embryos were mounted in $3 \%$ methyl cellulose solution (Fisher Scientific, Hampton, $\mathrm{NH}$ ) to orient them and were imaged in the continued presence of $2 \mathrm{mM}$ tricaine.

\section{Genotyping using PCR and heteroduplex mobility assay (HMA)}

Genomic DNA from single embryos was used as the template in polymerase chain reactions (PCRs) with 2x Taq Master Mix (New England Biology, Ipswich, MA). Genomic DNA was obtained by placing single embryos in PCR tubes with $10 \mu \mathrm{L}$ of lysis solution containing Proteinase $\mathrm{K}$ and incubated at $55^{\circ} \mathrm{C}$ for 2 hours, followed by $95^{\circ} \mathrm{C}$ for 10 minutes to inactivate the proteinase $\mathrm{K}$. A small aliquot $(0.5 \mu \mathrm{L})$ of this solution was directly used as a template to amplify a region flanking each CRISPR target site (Supplemental Table 4). PCR products were analyzed using a heteroduplex mobility assay (HMA) for assessing the nuclease activity and detecting the presence of indels [32-35]. In brief, the amplicons were subjected to denaturation followed by slow renaturation to facilitate the formation of heteroduplexes using a thermocycler. These samples were resolved on 6-8\% polyacrylamide gels and the resulting mobility profiles used to infer the efficiency of CRISPRCas9 nuclease activity. 


\section{Targeted amplicon sequence analysis}

PCR amplicons obtained from single embryos were cloned into pCR4 using the TOPO-TA cloning kit (Invitrogen, Carlsbad, CA). Ten representative colonies picked from each plate were grown in $1.5 \mathrm{~mL}$ liquid cultures to isolate plasmid DNA. Plasmid DNA was sequenced with M13 F and R primers using the Sanger Method.

\section{Structural modeling}

Protein three-dimensional structure predictions of mature wild-type ndr2 (126 amino acids) and translated mutant proteins were made using I-TASSER with no constraints (https://zhanglab.ccmb.med.umich.edu/l-TASSER/) [36-38]; the mature ndr2 amino acid sequence was derived from the precursor protein (nodal-related 2 precursor, 501 amino acids, NCBI Accession: NP_624359.1) following the cleavage recognition sequence of RXRXXR [19, 23] and containing amino acid residues 376-501. Template-based threading and modeling were performed using the top 10 structure templates identified by the Local Meta-Threading-Server (LOMETS) [39], including bone morphogenetic protein-7 (PDB code: $1 \mathrm{~m} 4 \mathrm{uL}$ ), growth factor (PDB code: $5 \mathrm{vz} 3 \mathrm{~A}$ ), TGF- $\beta$ ligand-receptor complex (PDB code: 3qb4A), and nodal/BMP2 (PDB code: $4 \mathrm{n} 1 \mathrm{dA}$ ). Images were generated and aligned in the modeling package PyMOL v2.0 (http://www.pymol.org).

\section{Results}

\section{In vitro assay as a quality control step for nuclease activity}

An in vitro assay was employed to check for nuclease activity of the sgRNA-Cas9 RNP complexes (Fig. 2A). Guides C-1, C-9, and C-12 did not show any cleavage products suggesting a lack of any nuclease activity. This could be due to degraded or poor sgRNA quality. The extent of nuclease activity for the remaining 12 guides was distinctly visible. The cleavage products correspond to the predicted cut sites in the PCR amplicons. For example, C-2 and C-3 had cut sites in Exon 1 within the same PCR amplicon. Each amplicon had different size cleavage products with C-2 yielding cleavage products of $194 \mathrm{bp}$ and $267 \mathrm{bp}$ and C-3 yielding cleavage products of $146 \mathrm{bp}$ and $315 \mathrm{bp}$ (Fig. 2A). These in vitro results showed that $12 / 15$ (80 \%) sgRNA guides showed noticeable nuclease activity, providing confidence that they were biochemically active to create double stranded breaks (DSBs) at the target site in the genome of a zebrafish embryo. The GC \% of these 12 sgRNA guides that showed clear nuclease activity in vitro ranged from 40-85\% (seven were between $40 \%$ and $55 \%$, six were $60-65 \%$, one was $70 \%$ and 1 was $85 \%$ ). None of the guides had a native 5' GG start, three had a GN start, and four had an NG start. The length of the guides 
(21- or 22-mers) and the absence of a native GG sequences did not appear to impact the in vitro nuclease activity.

\section{Phenotypic and genotypic analysis of $\mathrm{G}_{0}$ embryos injected with sgRNA-Cas9 RNP complexes}

Phenotypic analysis showed that 4/12 CRISPR/sgRNAs (33 \%) produced penetrant cyclopic phenotypes in 3 days post fertilization (dpf) zebrafish embryos (Fig. 3). Cyclopic mutants exhibited variably fused eyes from mild (Fig. 3B-C) to severe phenotypes (Fig. 3DE).

Genotypic analysis using PCR-HMA indicated that 4/12 CRISPR/sgRNAs (33 \%) caused mutations in single embryos that were analyzed (Fig. 2B, C, D, F; Supplemental Table 2). Indels could not be detected using HMA profile with the remaining eight CRISPR/sgRNAs (Fig. 2E \& G). There were two instances when the HMA profile did not validate the observed cyclopic phenotype. CRISPR/sgRNA C-2 yielded positive HMA results with no observed cyclopic phenotype (Fig. 2B, Supplemental Table 2), whereas CRISPR/sgRNA C-16 was negative for the HMA profile with a penetrant cyclopic phenotype (Fig. 2G, Supplemental Table 2). An additional round of injections on 32 embryos of the CRISPR/sgRNA C-3 revealed a similar discordant finding between phenotypic and genotypic analyses. Phenotypic analysis showed 2/32 (6 \%) injected embryos displaying cyclopic phenotypes. Upon analyzing the same embryos using PCR-HMA, 32/32 (100\%) injected embryos suggested the presence of indels indicated by heteroduplexes (Supplemental Fig 1). Altogether, this suggests that the level of mosaicism and functional outcome of each unique mutation impacts the penetrance and severity of the cyclopic phenotype.

To test the correlation between predicted on-target scores and in vivo nuclease activity, we selected sgRNAs with Doench (on-target) scores ranging from 3-77 (Supplemental Table 2). With respect to both the cyclopic phenotype and the nuclease activity detected using PCR-HMA, three of the five active CRISPR/sgRNAs (C-2, C-3, C-7) had Doench (on-target) scores of 63, 62, and 69, respectively. The remaining two active CRISPR/sgRNAs (C-13, C-16) had much lower predictive scores at 19 and 27.9. There were six CRISPR/sgRNAs with predicted scores higher than 30 that did not yield activity (Supplemental Table 2) suggesting that on-target guide scores are not always predictive of in vivo nuclease activity.

\section{Mutations causing loss-of-function phenotypes}

Multiple clones from PCR amplicons showing HMA profiles and/or cyclopic phenotypes were Sanger-sequenced to identify the genetic lesions. We obtained 3-6 mutant alleles for the CRISPR target regions (Table 1). Most mutant alleles identified across single 
embryos from five CRISPR/sgRNAs included small indels, in-frame indels, and complex indels, ranging from 1 to 30 base pairs. There were three larger deletions identified ranging from 71 to 172 base pairs. The majority of the identified mutations, 16/22 (73\%), resulted in frameshifts and predicted premature protein truncation that can explain the loss-of-function phenotypes.

As mentioned previously, there were two instances when the HMA profile did not match the observed cyclopic phenotype. For CRISPR/sgRNA C-2 that yielded positive HMA results with no observed cyclopic phenotype, four unique mutant alleles were confirmed with Sanger sequencing. Three of the four mutant alleles caused frameshifts and predicted lossof-function. With no observable cyclopic phenotype, this suggests that zebrafish embryos injected with C-2 possibly had low contribution of these loss-of-function mutant alleles as compared to the fourth detected in-frame deletion and/or remaining wild-type alleles. For CRISPR/sgRNA C-16 that yielded negative HMA indel profile with a penetrant cyclopic phenotype, three unique mutant alleles were confirmed with Sanger sequencing. One of the three mutant alleles was a 1-bp deletion predicted to cause loss-of-function while the other two yielded in-frame indels. With no observable positive HMA profile and penetrant cyclopic phenotype, this suggests that the zebrafish embryos injected with $\mathrm{C}-16$ possibly had a high contribution of the 1-bp deletion mutant allele that was difficult to resolve using PCR-HMA. These divergent findings from the HMA profile and observed cyclopic phenotypes illustrate the mosaicism of the $\mathrm{G}_{0}$ embryos.

\section{Importance of the "cysteine knot" in ndr2 structure-function}

In order to study the structure-function relationship of ndr2 and the guide C-16 mutants, we modeled three-dimensional (3D) structures of a predicted mature ndr2 ligand of 126 amino acids and identified mutant alleles generated from targeting exon 3 (guide C-16) (Fig. 4). I-TASSER provided the following amino acid sequence identities between the mature wild-type ndr2 ligand and corresponding threading templates utilized: $38 \%$ for bone morphogenetic protein-7 (PDB code: 1m4uL), $25 \%$ for growth factor (PDB code: 5vz3A), 42 $\%$ for TGF- $\beta$ ligand-receptor complex (PDB code: 3qb4A), and $62 \%$ for nodal/BMP2 (PDB code: $4 \mathrm{n} 1 \mathrm{dA}$ ). I-TASSER provided five models for each simulated protein and the top model was selected for further analysis. The I-TASSER confidence score (C-score) for model 1 of the mature wild-type ndr2 ligand was -0.60 with an estimated template modeling score (TMscore) of $0.64 \pm 0.13$. As observed, the simulated $3 D$ structures of mature wild-type ndr2 exhibited the common elements in the structure of transforming growth factor type $\beta$ superfamily factors, with five main $\beta$-sheets stretching outward structurally from the centrally located "cysteine knot" (Fig. 4A). 
The three identified mutant alleles generated from targeting exon 3 (guide C-16) yielded two in-frame indels (models $1-1$ and 1-2) and a frameshift deletion (model 2-1). The I-TASSER C-score for the mutant allele 1-1 model was -0.49 with an estimated TM-score of 0.65 . The mutant allele 1-1 was identified as a $3 \mathrm{bp}$ in-frame insertion. When we overlaid the protein structures of the mature wild-type ndr2 ligand and the mutant allele 1-1, the overall structure of these two proteins aligned very well with a global root-mean-square deviation (RMSD) of 1.188. The resulting mutant amino acid residue is predicted to disrupt two of the $\beta$-sheets resulting in them being smaller than in the wild type and structurally different (Fig. 4B).

The I-TASSER C-score for the mutant allele 1-2 model was -0.65 with an estimated TM-score of 0.63 . The mutant allele 1-2 was identified as a $9 \mathrm{bp}$ in-frame deletion. When we overlaid the protein structures of the mature wild-type ndr2 ligand and the mutant allele 1-2, the overall structure of these two proteins aligned very well with a global root-mean-square deviation (RMSD) of 1.383 . The loss of three amino acid residues is predicted to disrupt all $\beta$-sheets resulting in two different lengths compared to the wild type (Fig. 4C).

The I-TASSER C-score for the mutant allele 2-1 model was -2.65 with an estimated TM-score of 0.41 . The mutant allele $2-1$ was identified as a 1 bp deletion leading to a frameshift resulting in 65 mutant amino acid residues before terminating the reading frame. When we overlaid the protein structures of the mature wild-type ndr2 ligand and the mutant allele 2-1, structural differences were observed and the RMSD is 1.394. Two native cysteine residues were deleted and one mutant cysteine residue appeared due to this frameshift (Fig. 4D). The mutant cysteine residue was predicted to localize in the "cysteine knot" region and could be functional, but one cysteine residue was still missing. In addition, differences in the $\beta$-sheets mentioned above were present. Further, the additional mutant residues formed a long loop that appeared to be highly exposed to solvent. This long loop also sits very close to the cysteine knot.

All identified mutant alleles generated from targeting exon 3 were predicted to cause structural changes to the mature ndr2 ligand that could potentially impact function. Because the position of the native cysteine residues in forming the "cysteine knot" is essential for protein function, we suggest that the mutations we obtained with guide $\mathrm{C}-16$ result were either hypomorphic or null alleles.

\section{Discussion}

Most of the previous mutant alleles of the $n d r 2$ gene in zebrafish were generated in forward genetic screens with ENU mutagenesis making them difficult to characterize completely at the molecular level (Supplemental Table 1). Using programmable nucleases, 
especially the CRISPR-Cas9 system, there is a surge of mutant alleles for several genes that can be very well characterized. We tested the efficiency of nuclease activity of CRISPR/sgRNAs with Streptococcus pyogenes Cas9 and generated several mutant alleles in the zebrafish ndr2 gene.

Using a cloning-free method to generate T7-RNA polymerase-driven in vitro transcribed sgRNA (modified from [31]) and an in vitro nuclease assay, we learned that the lack of native GG dinucleotide at the beginning of the CRISPR guide is not essential for synthesis. As reported earlier, we also observe that guides that are 20-22 nt long can be effective. This is also true with respect to nuclease activity, both in vitro and in vivo. The in vitro nuclease activity is useful as a quality control step prior to embryo injections. However, the presence of in vitro nuclease activity did not always translate to in vivo activity in embryos. Based on these observations though, we find that the number of usable guides can be more than just the sites that start with canonical GG or GN/NG. In addition, the predicted on-target scores of our sample of CRISPR guides did not always correspond to observed activity in the embryos. This suggests that the current prediction scores for ontarget efficiency, especially in the context of zebrafish, need to be further improved using validation experiments. Ultimately, in vivo validation of CRISPR guides is necessary.

While Cas 9 was delivered as capped mRNA in earlier studies, an increasing number of studies use the Cas9 protein complexed with the guide RNA. Our study validates the effective use of RNP complexes for zebrafish embryo injections, which result in penetrant phenotypes. We were able to observe variable fusion of eyes across injected embryos.

This study also showcases the importance of the "cysteine knot" being essential for biological activity of the ndr2 protein. Even with removal of two native cysteines and the addition of a mutant cysteine in exon 3, loss-of-function and cyclopic phenotypes occurred. Targeting exon 3 , we found mutations at the C-terminus of $n d r 2$ leading to loss of protein function thereby causing cyclopia in zebrafish embryos.

\section{Data Availability Statement}

All relevant data are within the paper and its supporting information files.

\section{Funding}

This work was supported by a Teaching Innovation Grant to AKC by the Quality Enhancement Program (QEP) in the Center for Teaching \& Learning, and the Department of Genetics, University of Alabama at Birmingham (UAB). This study was part of a Coursebased Undergraduate Research Experience (CURE) for first year undergraduate students in the Science and Technology Honors (STH) Program at UAB. The STH Program provided support for materials and reagents. 


\section{Competing Interests}

The authors have declared that no competing interests exist.

\section{Supplementary Materials}

Supplemental Tables (1-4); Supplemental Figure 1

\section{Acknowledgements}

The authors thank the staff of the UAB Zebrafish Research Facility for providing care and maintenance of the facility, Dr. Robert Kesterson and the UAB Transgenic \& Genetically Engineered Models (TGEMs) Core facility for support, Dr. Brad Yoder (CDIB, UAB) for providing laboratory space, Dr. Michael Miller (CDIB, UAB) for help with microscopy, Dr. Ben Johnson (VAI) and Dr. David Crossman (Genetics, UAB) for help with sequence analysis, Kartik Manne for advice on structure prediction using PyMol, Ansuya Jogi and Dr. Kiranam Chatti for editing and proofreading, and the administrative support of the Science and Technology Honors (STH) Program. Thanks to Dr. Oreoluwa Adedoyin (MERIT Postdoctoral Scholar) and Lindsay Jenkins (STH undergraduate student) for assistance in the course. AKC thanks Dr. Diane Tucker (STH Program) for advice on designing the CURE.

\section{Author Contributions}

Conceived and designed the experiments: ANT, RSA, IEB, LNB, JCD, DMG, JPG, BAH, ASK, JBM, VLM, ZPM, CLN, RKP, MCR, YAS, APW, SRG, SDF, AKC

Performed the experiments: ANT, RSA, IEB, LNB, JCD, DMG, JPG, BAH, ASK, JBM, VLM, ZPM, CLN, RKP, MCR, YAS, APW, SRG, SDF, AKC

Data analysis: ANT, RSA, IEB, LNB, JCD, DMG, JPG, BAH, ASK, JBM, VLM, ZPM, CLN, RKP, MCR, YAS, APW, SRG, SDF, AKC

Contributed reagents/materials/analysis tools: ANT, RSA, IEB, LNB, JCD, DMG, JPG, BAH, ASK, JBM, VLM, ZPM, CLN, RKP, MCR, YAS, APW, SRG, SDF, AKC

Manuscript preparation: ANT, AKC 


\section{References}

1. Massague, J., The transforming growth factor-beta family. Annu Rev Cell Biol, 1990. 6: p. 597-641.

2. Kingsley, D.M., The TGF-beta superfamily: new members, new receptors, and new genetic tests of function in different organisms. Genes Dev, 1994. 8(2): p. 133-46.

3. Rebagliati, M.R., et al., Zebrafish nodal-related genes are implicated in axial patterning and establishing left-right asymmetry. Dev Biol, 1998. 199(2): p. 261-72.

4. Rebagliati, M.R., et al., cyclops encodes a nodal-related factor involved in midline signaling. Proc Natl Acad Sci U S A, 1998. 95(17): p. 9932-7.

5. Feldman, B., et al., Nodal-related signals establish mesendodermal fate and trunk neural identity in zebrafish. Curr Biol, 2000. 10(9): p. 531-4.

6. Whitman, M., Nodal signaling in early vertebrate embryos: themes and variations. Dev Cell, 2001. 1(5): p. 605-17.

7. Dougan, S.T., et al., The role of the zebrafish nodal-related genes squint and cyclops in patterning of mesendoderm. Development, 2003. 130(9): p. 1837-51.

8. Schier, A.F., et al., Mutations affecting the development of the embryonic zebrafish brain. Development, 1996. 123: p. 165-78.

9. Roessler, E., et al., Cumulative ligand activity of NODAL mutations and modifiers are linked to human heart defects and holoprosencephaly. Mol Genet Metab, 2009. 98(12): p. 225-34.

10. Sampath, K., et al., Induction of the zebrafish ventral brain and floorplate requires cyclops/nodal signalling. Nature, 1998. 395(6698): p. 185-9.

11. Schier, A.F. and M.M. Shen, Nodal signalling in vertebrate development. Nature, 2000. 403(6768): p. 385-9.

12. Hatta, K., et al., The cyclops mutation blocks specification of the floor plate of the zebrafish central nervous system. Nature, 1991. 350(6316): p. 339-41.

13. Kimmel, C.B., T.F. Schilling, and K. Hatta, Patterning of body segments of the zebrafish embryo. Curr Top Dev Biol, 1991. 25: p. 77-110.

14. Hatta, K., A.W. Puschel, and C.B. Kimmel, Midline signaling in the primordium of the zebrafish anterior central nervous system. Proc Natl Acad Sci U S A, 1994. 91(6): p. 2061-5.

15. Halpern, M.E., et al., Genetic interactions in zebrafish midline development. Dev Biol, 1997. 187(2): p. 154-70.

16. Talbot, W.S., et al., Genetic analysis of chromosomal rearrangements in the cyclops region of the zebrafish genome. Genetics, 1998. 148(1): p. 373-80.

17. Tian, J., et al., A temperature-sensitive mutation in the nodal-related gene cyclops reveals that the floor plate is induced during gastrulation in zebrafish. Development, 2003. 130(14): p. 3331-42.

18. Lim, S., et al., A simple strategy for heritable chromosomal deletions in zebrafish via the combinatorial action of targeting nucleases. Genome Biol, 2013. 14(7): p. R69.

19. Constam, D.B., Regulation of TGFbeta and related signals by precursor processing. Semin Cell Dev Biol, 2014. 32: p. 85-97.

20. Kunnapuu, J., I. Bjorkgren, and O. Shimmi, The Drosophila DPP signal is produced by cleavage of its proprotein at evolutionary diversified furin-recognition sites. Proc Natl Acad Sci U S A, 2009. 106(21): p. 8501-6.

21. Jing, X.H., et al., Mechanisms underlying long- and short-range nodal signaling in Zebrafish. Mech Dev, 2006. 123(5): p. 388-94.

22. Remacle, A.G., et al., Substrate cleavage analysis of furin and related proprotein convertases. A comparative study. J Biol Chem, 2008. 283(30): p. 20897-906.

23. Nelsen, S.M. and J.L. Christian, Site-specific cleavage of BMP4 by furin, PC6, and PC7. J Biol Chem, 2009. 284(40): p. 27157-66.

24. Mesnard, D. and D.B. Constam, Imaging proprotein convertase activities and their regulation in the implanting mouse blastocyst. J Cell Biol, 2010. 191(1): p. 129-39. 
25. McDonald, N.Q. and W.A. Hendrickson, A structural superfamily of growth factors containing a cystine knot motif. Cell, 1993. 73(3): p. 421-4.

26. Massague, J., TGF-beta signal transduction. Annu Rev Biochem, 1998. 67: p. 75391.

27. Daopin, S., et al., Crystal structure of transforming growth factor-beta 2: an unusual fold for the superfamily. Science, 1992. 257(5068): p. 369-73.

28. Mason, A.J., Functional analysis of the cysteine residues of activin A. Mol Endocrinol, 1994. 8(3): p. 325-32.

29. Hsu, P.D., et al., DNA targeting specificity of RNA-guided Cas9 nucleases. Nat Biotechnol, 2013. 31(9): p. 827-32.

30. Doench, J.G., et al., Optimized sgRNA design to maximize activity and minimize offtarget effects of CRISPR-Cas9. Nat Biotechnol, 2016. 34(2): p. 184-191.

31. Gagnon, J.A., et al., Efficient mutagenesis by Cas9 protein-mediated oligonucleotide insertion and large-scale assessment of single-guide RNAs. PLoS One, 2014. 9(5): p. e98186.

32. Jinek, M., et al., A programmable dual-RNA-guided DNA endonuclease in adaptive bacterial immunity. Science, 2012. 337(6096): p. 816-21.

33. Jao, L.E., S.R. Wente, and W. Chen, Efficient multiplex biallelic zebrafish genome editing using a CRISPR nuclease system. Proc Natl Acad Sci U S A, 2013. 110(34): p. 13904-9.

34. Hwang, W.Y., et al., Efficient genome editing in zebrafish using a CRISPR-Cas system. Nat Biotechnol, 2013. 31(3): p. 227-9.

35. Challa, A.K., et al., Novel Hypomorphic Alleles of the Mouse Tyrosinase Gene Induced by CRISPR-Cas9 Nucleases Cause Non-Albino Pigmentation Phenotypes. PLoS One, 2016. 11(5): p. e0155812.

36. Yang, J., et al., The I-TASSER Suite: protein structure and function prediction. Nat Methods, 2015. 12(1): p. 7-8.

37. Roy, A., A. Kucukural, and Y. Zhang, I-TASSER: a unified platform for automated protein structure and function prediction. Nat Protoc, 2010. 5(4): p. 725-38.

38. Zhang, Y., I-TASSER server for protein 3D structure prediction. BMC Bioinformatics, 2008. 9: p. 40.

39. Wu, S. and Y. Zhang, LOMETS: a local meta-threading-server for protein structure prediction. Nucleic Acids Res, 2007. 35(10): p. 3375-82. 
bioRxiv preprint doi: https://doi.org/10.1101/277715; this version posted August 25, 2018. The copyright holder for this preprint (which was not certified by peer review) is the author/funder, who has granted bioRxiv a license to display the preprint in perpetuity. It is made available under aCC-BY 4.0 International license.

Table 1. Mutations in $n d r 2$ induced using sgRNA-Cas9 RNP complexes

\begin{tabular}{|c|c|c|c|}
\hline ID (HMA) & Sequence $\left(5^{\prime}-3^{\prime}\right)$ & Indels & Protein \\
\hline \multirow{2}{*}{ EXON $1 \mathrm{C}-2$ WT } & CACCGCATGCATCTGCCCACGTACATGATGCATCTCTATCGGCATTACAAGATGAACCAGACGCGCATACCCGCGGAGAGCCTCGAGCACGAGCACGCAGAC & & \\
\hline & 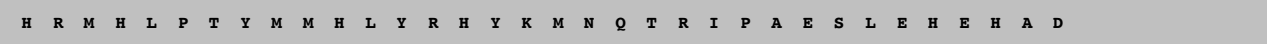 & & \\
\hline \multirow[t]{2}{*}{ c-2 $1-1$} & CACCGCATGCATCTGCCCACG---ATGATGCATCTCTATCGGCATTACAAGATGAACCAGACGCGCATACCCGCGGAGAGCCTCGAGCACGAGCACGCAGAC & $-3 \mathrm{bp}$ & $\begin{array}{l}\text { In-frame } \\
\text { deletion }\end{array}$ \\
\hline & 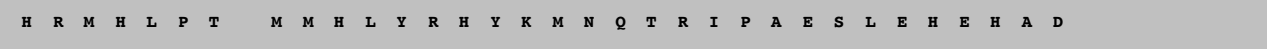 & 1 aa del & \\
\hline \multirow{2}{*}{ c-2 $1-2$} & CACCGCATGCATCTGCCCACGT----GATGCATCTCTATCGGCATTACAAGATGAACCAGACGCGCATACCCGCGGAGAGCCTCGAGCACGAGCACGCAGAC & $-4 \mathrm{bp}$ & Deletion \\
\hline & 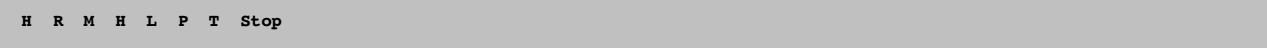 & Stop & \\
\hline \multirow{2}{*}{ c-2 $1-3$} & CACCGCATGCATCTGCCCACGTAACATGATGCATCTCTATCGGCATTACAAGATGAACCAGACGCGCATACCCGCGGAGAGCCTCGAGCACGAGCACGCAGAC & $+1 \mathrm{bp}$ & Insertion \\
\hline & 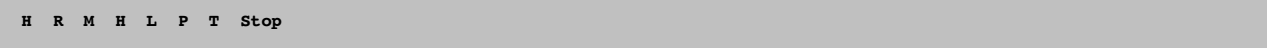 & Stop & \\
\hline \multirow[t]{2}{*}{ C-2 $2-1$} & CACCGCATGCATCTGCCCAC-- (-172) --GTGCTCAACAATGACAACCACTACGTGGCGATCTTTGACCTGTCTCCGGTTTTGTCGGAACGTCA & -172 bp & Deletion \\
\hline & 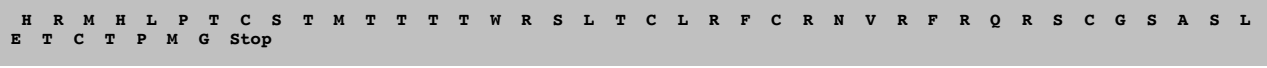 & $\begin{array}{l}40 \text { aa* }^{*} \text { ins }+ \\
\text { stop }\end{array}$ & \\
\hline \multirow[t]{2}{*}{ EXON 1 C-3 WT } & CACCGCATGCATCTGCCCACGTACATGATGCATCTCTATCGGCATTACAAGATGAACCAGACGCGCATACCCGCGGAGAGCCTCGAGCACGAGCACGCAGAC & & \\
\hline & 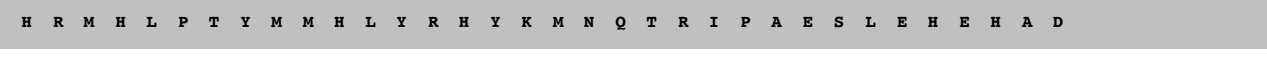 & & \\
\hline \multirow[t]{2}{*}{ c-3 $1-1$} & $\begin{array}{l}\text { CACCGCATGCATCTGCCCACGTACATGAGGCATCTCTATCGGCATTACAAGATGAACCAGACGCGC-------- } \\
\text { GGAGAGCCTCGAGCACGAGCACGCAGACACAATCAGGAGCATCATGTCTAAA }\end{array}$ & $-8 \mathrm{bp}$ & Deletion \\
\hline & 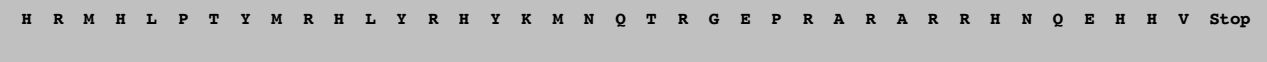 & $\begin{array}{l}16 a^{*} \text { ins }+ \\
\text { stop }\end{array}$ & \\
\hline \multirow[t]{2}{*}{ c-3 2-1 } & $\begin{array}{l}\text { CACCGCATGCATCTGCCCACGTACATGATGCATCTCTATCGGCATTACAAGATGAACCAGACGCGCATA-------- } \\
\text { GAGCCTCGAGCACGAGCACGCAGACACAATCAGGAGCATCATGTCTAAA }\end{array}$ & $-8 \mathrm{bp}$ & Deletion \\
\hline & 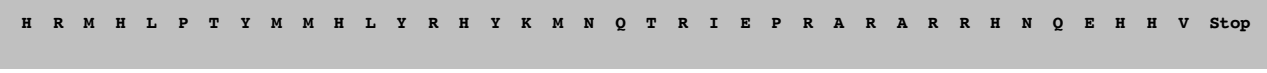 & $\begin{array}{l}15 \text { aa* ins }+_{\text {stop }}\end{array}$ & \\
\hline \multirow[t]{2}{*}{$c-32-2$} & $\begin{array}{l}\text { CACCGCATGCATCTGCCCACGTACATGATGCATCTCTATCGGCATTACAAGATGAACCAGACGCGCATAC------- } \\
\text { GAGCCTCGAGCACGAGCACGCAGACACAATCAGGAGCATCATGTCTAAA }\end{array}$ & $-7 \mathrm{bp}$ & Deletion \\
\hline & 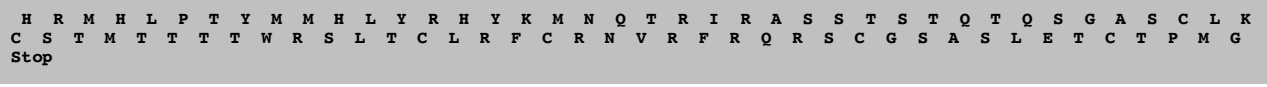 & $\begin{array}{l}57 a^{*} \text { ins }+ \\
\text { stop }\end{array}$ & \\
\hline \multirow[t]{2}{*}{$c-32-3$} & $\begin{array}{l}\text { CACCGCATGCATCTGCCCACGTACATGATGCATCTCTATCGGCATTACAAGATGAACCAGACGCGCAT-- } \\
\text { CCGCGAGAGCCTCGAGCACGAGCACGCAGACACAATCAGGAGCATCATGTCTAAA }\end{array}$ & -2 bp & Deletion \\
\hline & 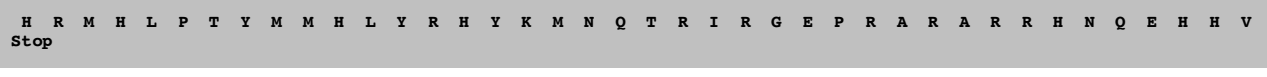 & $\begin{array}{l}17 a^{*} \text { ins }+ \\
\text { stop }\end{array}$ & \\
\hline \multirow[t]{2}{*}{$c-32-4$} & $\begin{array}{l}\text { CACCGCATGCATCTGCCCACGTACATGAGGCATCTCTATCGGCATTACAAGATGAACCAGACGCGC-------- } \\
\text { GGAGAGCCTCGAGCACGAGCACGCAGACACAATCAGGAGCATCATGTCTAAA }\end{array}$ & $-8 \mathrm{bp}$ & Deletion \\
\hline & 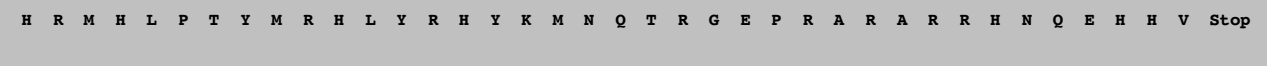 & $\begin{array}{l}16 \text { aa* ins }+ \\
\text { stop }\end{array}$ & \\
\hline \multirow[t]{2}{*}{ EXON $1 \mathrm{C}-7$ WT } & CACCGCATGCATCTGCCCACGTACATGATGCATCTCTATCGGCATTACAAGATGAACCAGACGCGCATACCCGCGGAGAGCCTCGAGCACGAGCACGCAGAC & & \\
\hline & 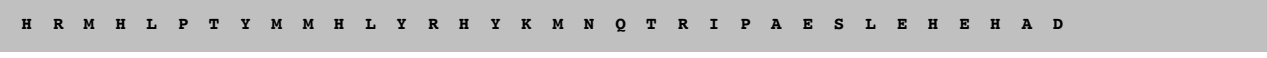 & & \\
\hline \multirow[t]{2}{*}{ c-7 $1-1$} & $\begin{array}{l}\text { CACCGCATGCATCTGCCCAC---- } \\
\text { ATGATGCATCTCTATCGGCATTACAAGATGAACCAGACGCGCATACCCGCGGAGAGCCTCGAGCACGAGCACGCAGACACAATCAGGAGCATCATGTCTAAA }\end{array}$ & -4 bp & Deletion \\
\hline & $\begin{array}{lllllllllll} & \text { H } & \text { R } & \text { M } & \text { H } & \text { L } & \text { P } & \text { T } & \text { Stop } & & \\
\end{array}$ & Stop & \\
\hline \multirow[t]{2}{*}{ c-7 1-2 } & 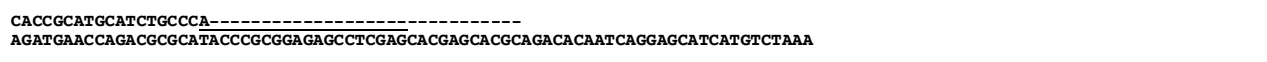 & $-30 \mathrm{bp}$ & $\begin{array}{l}\text { In-frame } \\
\text { deletion }\end{array}$ \\
\hline & 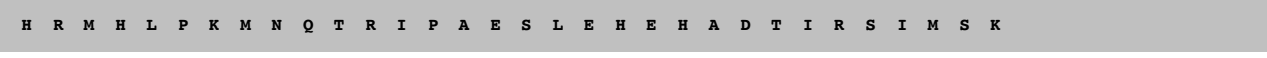 & 10 aa del & \\
\hline \multirow[t]{2}{*}{ C-7 $1-3$} & $\begin{array}{l}\text { CACCGCATGCATCTGCCCATTA-- } \\
\text { TACATGATGCATCTCTATCGGCATTACAAGATGAACCAGACGCGCATACCCGCGGAGAGCCTCGAGCACGAGCACGCAGACACAATCAGGAGCATCATGTCTAAA }\end{array}$ & $+3,-2$ bp & Complex indel \\
\hline & 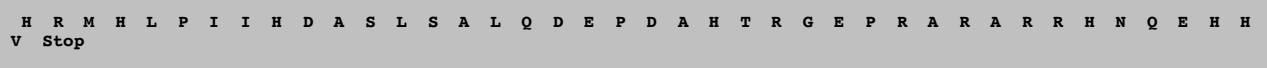 & $\begin{array}{l}35 \mathrm{aa}^{\star} \text { ins }+ \\
\text { stop }\end{array}$ & \\
\hline \multirow[t]{2}{*}{ c-7 2-1 } & $\begin{array}{l}\text { CACCGCATGCATCTGCCCACGT----- } \\
\text { GATGCATCTCTATCGGCATTACAAGATGAACCAGACGCGCATACCCGCGGAGAGCCTCGAGCACGAGCACGCAGACACAATCAGGAGCATCATGTCTAAA }\end{array}$ & -4 bp & Deletion \\
\hline & 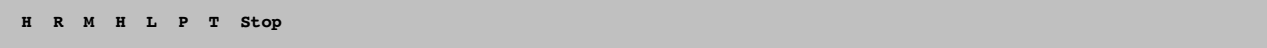 & Stop & \\
\hline EXON 2 C-12 WT & TGCAGGAGGGTGGACATGCATGTGGATTTTAACCAGATCGGATGGGGCTCCTGGATCGTGTTCCCTAAGAAGTACAATGCATACCGGTGCGAGGGGGCCTGC & & \\
\hline & 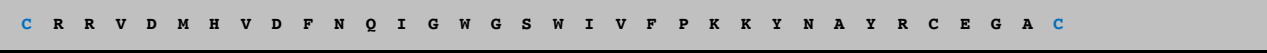 & & \\
\hline
\end{tabular}


bioRxiv preprint doi: https://doi.org/10.1101/277715; this version posted August 25, 2018. The copyright holder for this preprint (which was not certified by peer review) is the author/funder, who has granted bioRxiv a license to display the preprint in perpetuity. It is made available under aCC-BY 4.0 International license.

\begin{tabular}{|c|c|c|c|}
\hline C-12 1-1 & 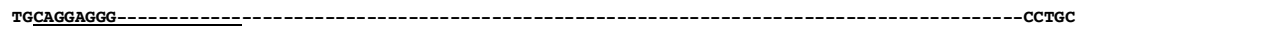 & $-87 \mathrm{bp}$ & $\begin{array}{l}\text { In-frame } \\
\text { deletion }\end{array}$ \\
\hline & 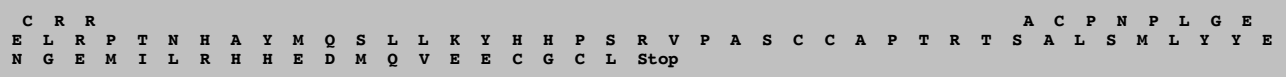 & 29 aa del & \\
\hline \multirow[t]{2}{*}{ C-12 $1-2$} & TGCAGGAGGGTGGACATG----TGGATTTTAACCAGATCGGATGGGGCTCCTGGATCGTGTTCCCTAAGAAGTACAATGCATACCGGTGCGAGGGGGCCTGC & $-4 \mathrm{bp}$ & Deletion \\
\hline & 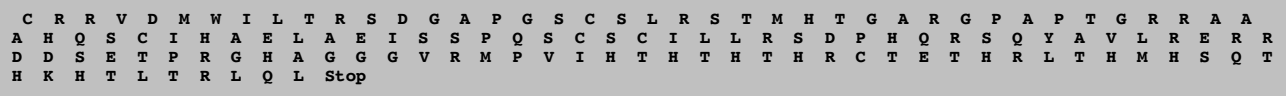 & $\begin{array}{l}126 \text { aa* ins }+ \\
\text { stop }\end{array}$ & \\
\hline \multirow[t]{2}{*}{ C-12 2-1 } & TGCAGGAGGGTGGACATGCGGACATGTGGATTTTAACCAGATCGGATGGGGCTCCTGGATCGTGTTCCCTAAGAAGTACAATGCATACCGGTGCGAGGGGGCCTGC & $+4 \mathrm{bp}$ & Insertion \\
\hline & $\begin{array}{llllllllllllllllll}C & R & R & V & D & M & R & T & C & G & F & \text { Stop } & & & & \end{array}$ & 5 aa* ins + stop & \\
\hline \multirow[t]{2}{*}{ C-12 2-2 } & 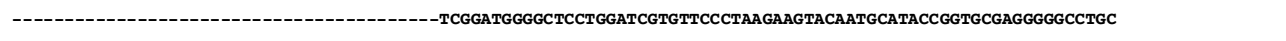 & $-71 \mathrm{bp}$ & Deletion \\
\hline & 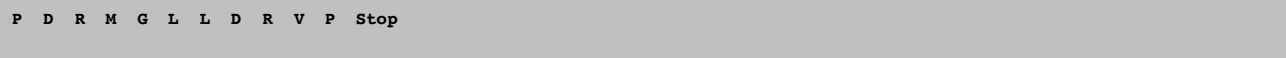 & $\begin{array}{l}10 \text { aa*ins }+ \\
\text { stop }\end{array}$ & \\
\hline \multirow[t]{2}{*}{$C-12 \quad 2-3$} & TGCAGGAGGGTGGACATCCACA-GTGGATTTTAACCAGATCGGATGGGGCTCCTGGATCGTGTTCCCTAAGAAGTACAATGCATACCGGTGCGAGGGGCCTGC & $1 \mathrm{sub},+2,-1 \mathrm{bp}$ & Complex indel \\
\hline & 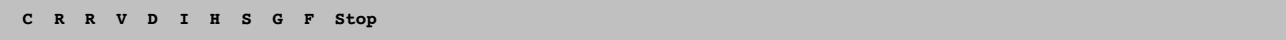 & 3 aa* ins + stop & \\
\hline \multirow[t]{2}{*}{ C-12 2-4 } & TGCAGGAGGGTGGA-------TTTTAACCAGATCGGATGGGGCTCCTGGATCGTGTTCCCTAAGAAGTACAATGCATACCGGTGCGAGGGGGCCTGC & -12 bp & $\begin{array}{l}\text { In-frame } \\
\text { deletion }\end{array}$ \\
\hline & 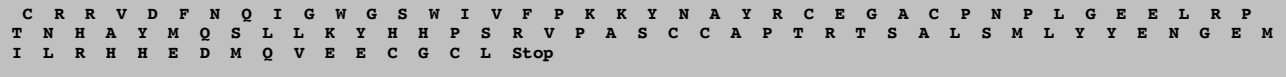 & 4 aa del & \\
\hline \multirow[t]{2}{*}{ EXON 3 C-16 WT } & $\begin{array}{l}\text { CATCACCCCAGTCGTGTTCCTGCATCCTGCTGCGCTCCGACCCGCACCAGCGCTCTCAGTATGCTGTACTACGAGAACGGAGAGATGATTCTGAGACACCACGAGGACATGCAGGTGGAGG } \\
\text { AGTGCGGATGCCTGTGA }\end{array}$ & & \\
\hline & 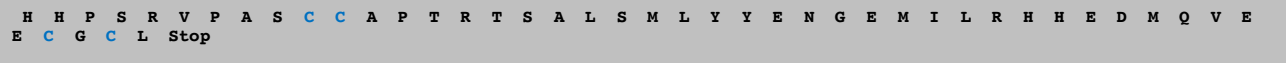 & & \\
\hline \multirow[t]{2}{*}{ c-16 1-1 } & $\begin{array}{l}\text { CATCACCCCAGTCGTGTTCCTGCATCCTGCTGCGCTCCGACCCGCACCAGCGCTCTCAGTATGCTGTACTACGAGAACGGAGAGATGATTCTGAGACACCACGAGGACATTCTGCAGGTGG } \\
\text { AGGAGTGCGGATGCCTGTGA }\end{array}$ & $+3 \mathrm{bp}$ & $\begin{array}{l}\text { In-frame } \\
\text { insertion }\end{array}$ \\
\hline & 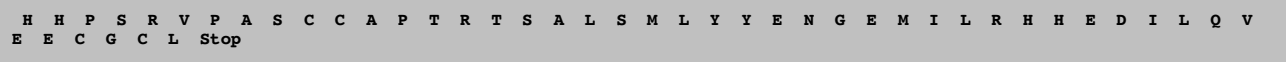 & $\begin{array}{l}1 \text { aa sub*, } 1 \text { aa* } \\
\text { ins }\end{array}$ & \\
\hline \multirow[t]{2}{*}{ C-16 1-2 } & $\begin{array}{l}\text { CATCACCCCAGTCGTGTTCCTGCATCCTGCTGCGCTCCGACCCGCACCAGCGCTCTCAGTATGCTGTACTACGAGAACGGAGAGATGATTCTGAGACAC-------- } \\
\text { ATGCAGGTGGAGGAGTGCGGTGCCTGTGA }\end{array}$ & $-9 \mathrm{bp}$ & $\begin{array}{l}\text { In-frame } \\
\text { deletion }\end{array}$ \\
\hline & 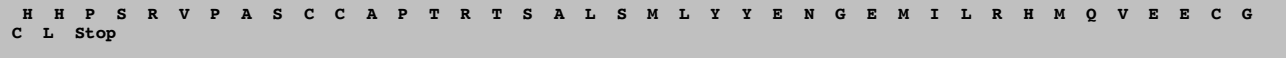 & 3 aa del & \\
\hline \multirow[t]{2}{*}{ C-16 2-1 } & $\begin{array}{l}\text { CATCACCCAGTCGTGTTCCTGCATCCTGCTGCGCTCCGACCCGCACCAGCGCTCTCAGTATGCTGTACTACGAGAACGGAGAGATGATTCTGAGACACCACGAGGACA- } \\
\text { GCAGGTGGAGGAGTGCGGATGCCTGTGA }\end{array}$ & -1 bp & Deletion \\
\hline & 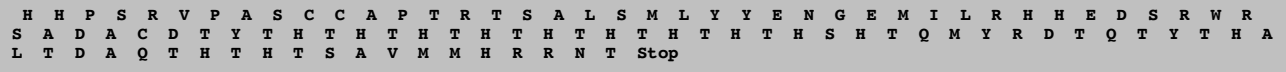 & $\begin{array}{l}65 \text { aa* ins }+ \\
\text { stop }\end{array}$ & \\
\hline
\end{tabular}


A.

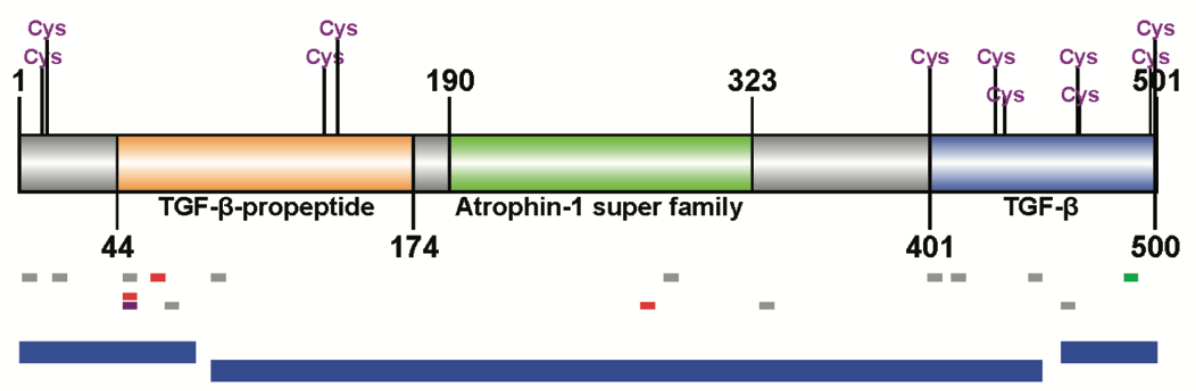

B.

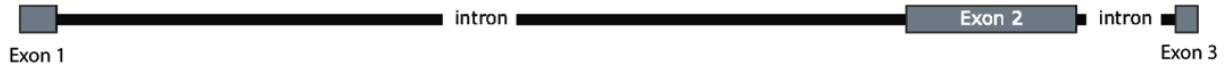

C.

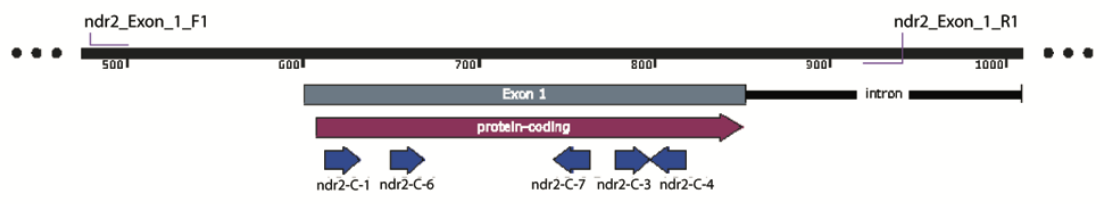

sine

$\rightarrow$

D.

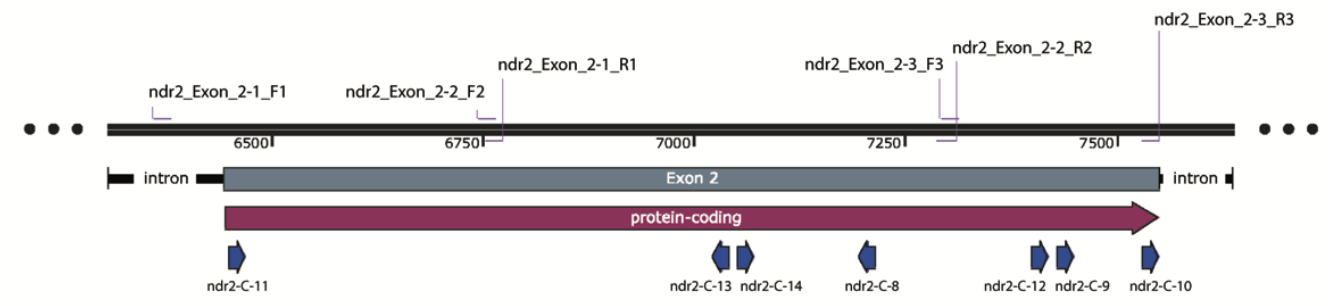

E.

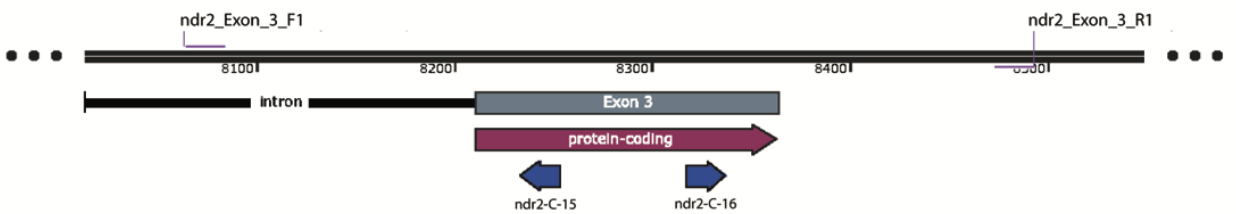

Figure 1. CRISPR targeting in the $n d r 2$ gene. (A) Schematic showing ndr2 protein structure along with CRISPR sgRNA guides that yielded negative HMA profile and phenotype (gray lines), positive HMA profile and negative phenotype (purple line), negative HMA profile and positive phenotype (green line), positive HMA profile and phenotype (red lines). The bottom blue regions correspond to exons of the ndr2 gene. (B) Schematic showing ndr2 gene structure (chr 12: 48,295,205 - 48,302,968). (C-E) Schematics for individual exons (exons 1-3) showing CRISPR targeting regions (blue arrows), PCR primer binding sites (light purple lines), and amplicon sizes. 

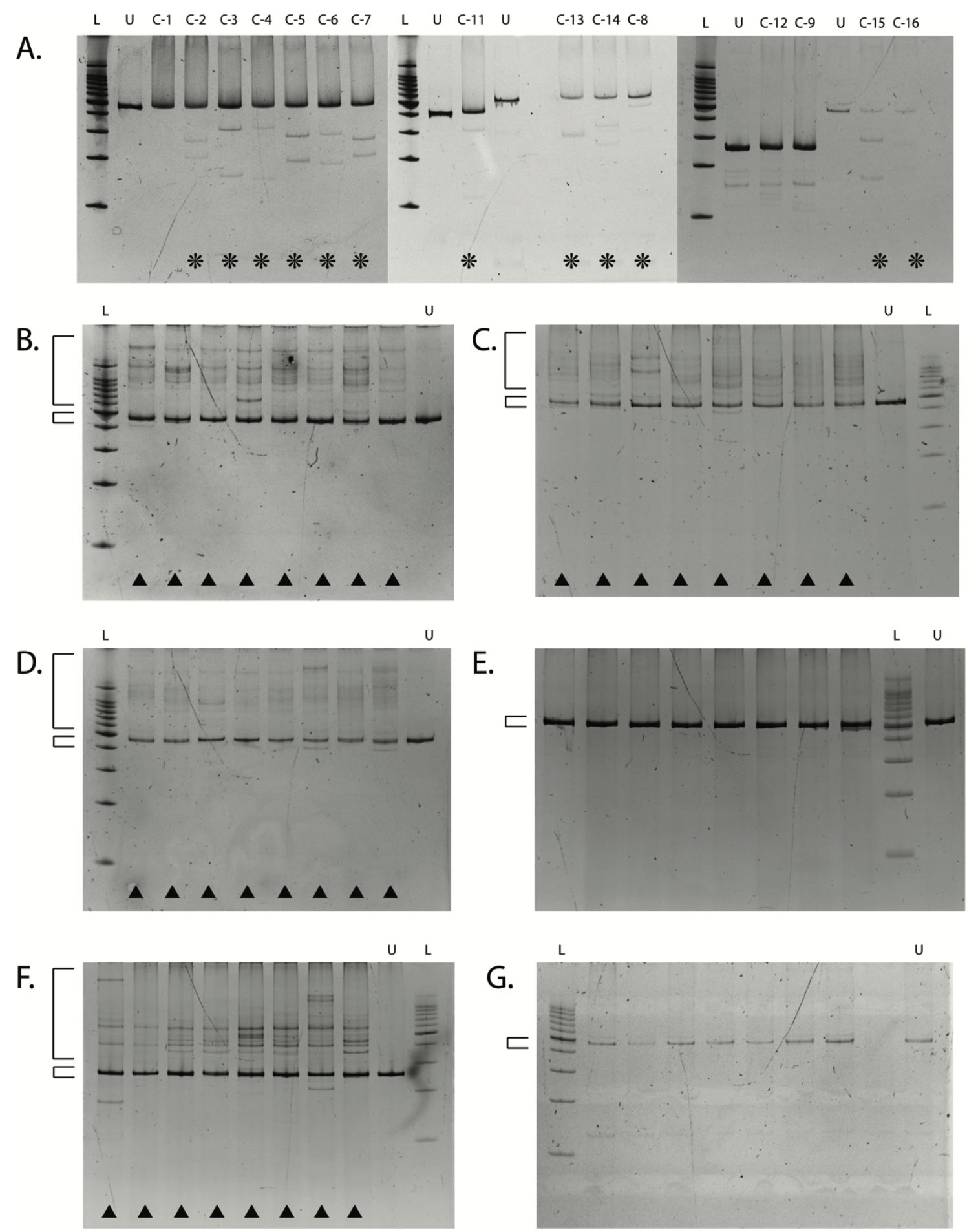

Figure 2. In vitro nuclease activity of sgRNA-Cas9 RNP complexes and indel detection in the ndr2 gene using heteroduplex mobility assay. (A) Images of ethidium bromide stained polyacrylamide gels (6\%) showing in vitro sgRNA-Cas9 RNP complex activity by cutting amplified CRISPR-targeted region (positive cutting, star) for all sgRNA. (B-G) Images of ethidium bromide stained polyacrylamide gels (6\%) showing separation of homoduplex and heteroduplex PCR amplicons from sgRNA-Cas9 RNP complexes injected into single zebrafish embryos (positive HMA, arrowheads). CRISPR targeting guide: C-2 (B), C-3 (C), C-7 (D), C-12 (E), C-13 (F), and C-16 (G). Small and large square brackets indicate homoduplex and heteroduplex bands, respectively. $L=100 \mathrm{bp}$ ladder, $\mathrm{U}=$ uncut amplified PCR product control or uninjected wild-type control. 

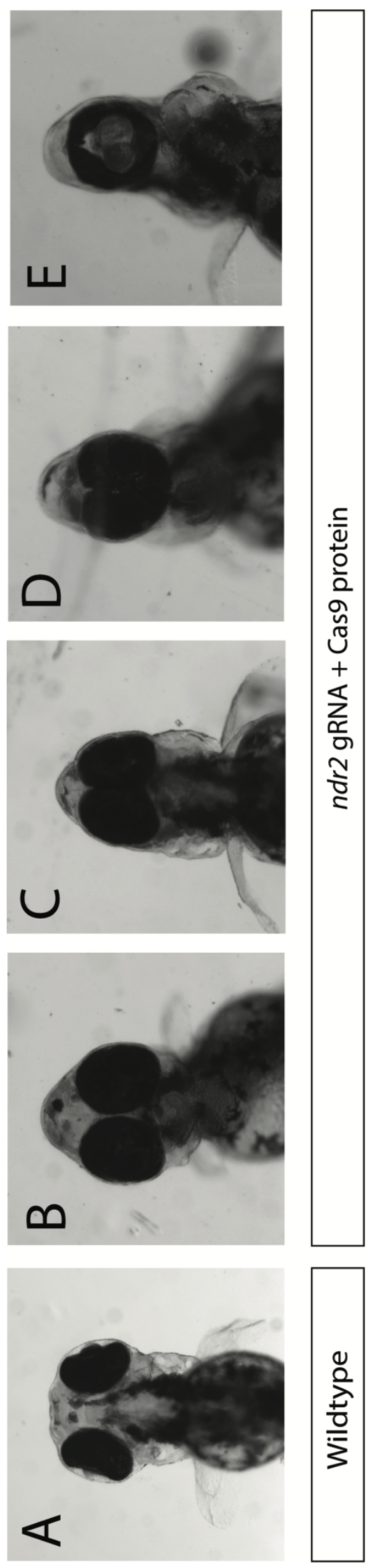

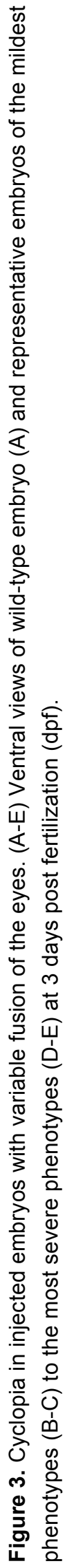


A

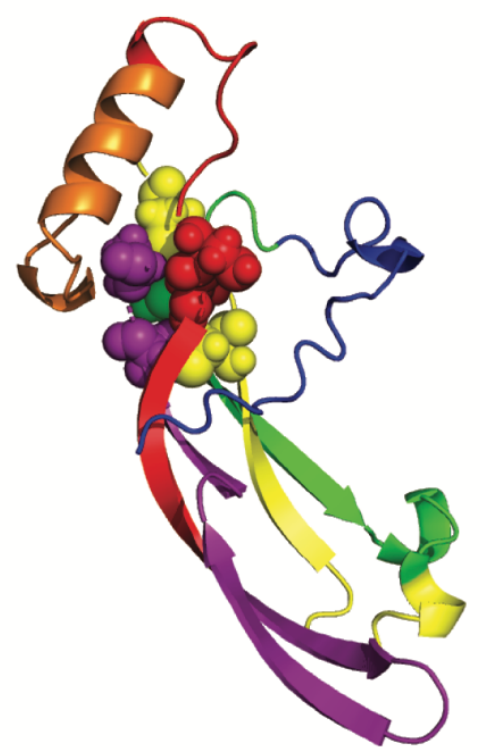

C

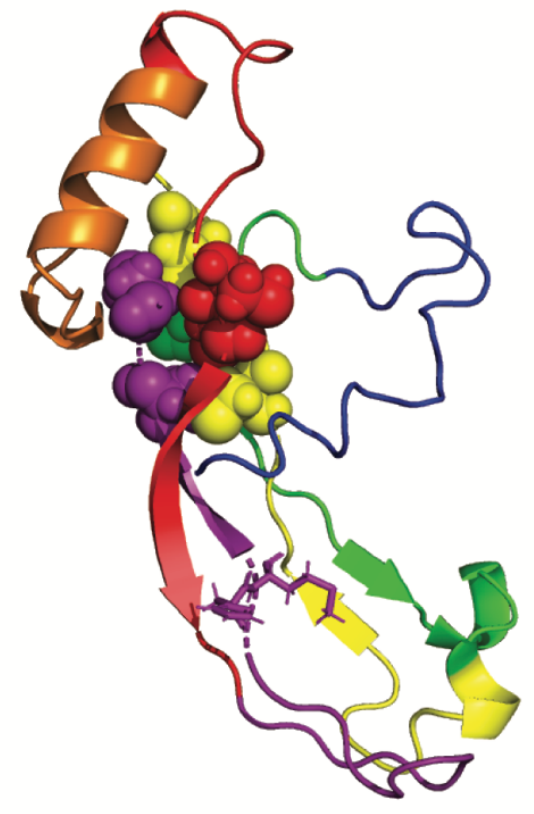

B

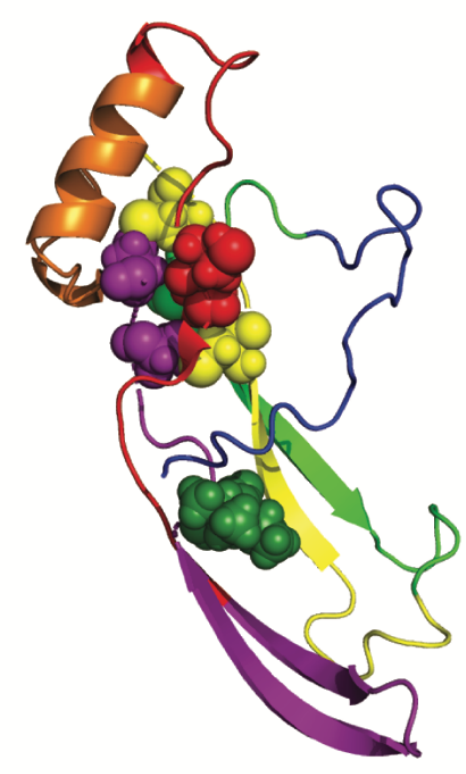

D

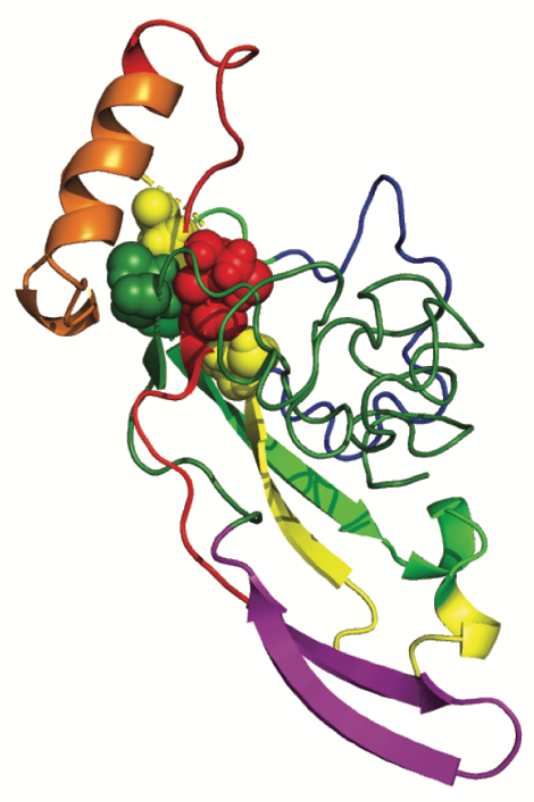

Figure 4. Predicted 3D structure of the mature wild-type ndr2 protein and the mutant alleles generated from targeting exon 3 (guide $C-16$ ). (A-D) The structures were generated with ndr2 protein sequence for mature wild type (A), mutant alleles 1-1 and 1-2 resulting in in-frame indels (B-C), and mutant allele 2-1 resulting in a 1 bp deletion (D). Depictions as follows: native cysteine residues are colored spheres (red, yellow, green and purple) (A-D), mutant amino acid residues are dark green spheres (B), two amino acid residues surrounding in-frame deletion are sticks $(C)$, and mutant amino acid residues are dark green ribbon with novel cysteine residue as dark green spheres (D). 